\title{
Structural Transactional Analysis: Ego Selves and Ego States - Cause-Effect and Interventions
}

\section{(C) 2021 Jorge Alberto Close}

Editor's Note: this article appeared first in Portuguese in Revista Brasiliera de Análise Transacional (REBAT) Ano XXX 2021 and is translated and re-published here with kind permission from that journal. The original version can be seen at https://unat.org.br/portal/rebat2021.php

"How are we to hope that, one day, we might free ourselves of the animal we carry within us, if we are never informed of how the wonderful mechanism called the nervous system works within us?"

Attributed to Henri Laborit (1914-1995).

\begin{abstract}
This article identifies the physiological, neurological, and psychological determinants that arise from constraints imposed by both genetic and environmental factors, originating human behaviours. The determinants, called Ego Selves, that organise the phenomena that Eric Berne classified, structured, conceptualised, and defined to mould transactional analysis and design instruments to assist professionals and patients to adjust behaviours, are analysed. A different form of presenting the adapted Child, differentiating it from Berne's model where the adapted Child is shown as a part of the natural Child, is presented. Parent-Adapted Child, and the Adult ego states, adjusting their manifestation and organization to the physiological development of their corresponding ego self, are identified, proposing that the Adapted Child is a part of the Parent ego state. Contamination is reviewed and adjusted for consistency between cause and effect, identifying that the contaminated ego state is the Parent ego state, creating a delusion based on injunctions that generate an illusion in the adapted Child portion of it which in turn causes the natural child's emotional reaction, considerably limiting the Adult ego state's capabilities to intervene. Script analysis is reviewed and organised indicating that the script is a life plan initiated at conception and ending at death, and that it is
\end{abstract}

indispensable for survival, having adequate and inadequate segments that may limit lifespan and quality of life. Occurrences, neurophysiological factors, and memories involved in their development and implementation are also identified. Suggestions and examples for the integrated development of intervention strategies and tactics to adjust behaviours and fulfil contracts are presented in the corresponding section.

\section{Keywords}

structural analysis; ego states; contamination, script; self; needs; homeostasis; behaviour; development stages; phylogenetic memories, ontogenetic memories

\section{Introduction}

Berne (1961) defined ego states as manifestations of three different psychic organs - the archeopsyche, the exteropsyche, and the neopsyche - alluding to but not identifying areas of the central nervous system. He also stated, "We shall now speak of three instances: determinants, organizers, and phenomena" (p. 264) (italics in original), going on to define determinants as "factors which determine the quality of the organization and the phenomena, that is, they establish their programming" (p. 265). This article will review the determinants that originate human behaviour and explore how these determinants, called Ego Selves, organise the phenomena that Berne classified, structured, and conceptualised to mould transactional analysis.

I will comment on:

- the Free Child, Parent-Adapted Child, and the Adult ego states relationship;

- contamination for consistency between cause and effect;

- script development and organisation to reflect neurophysiological factors involved in their development and functionality. 
I will include suggestions for the development of interactive intervention strategies.

Human beings are a development of the animal kingdom, and, considering the neurophysiological aspects, operate as Selves. The term Self is used to signify "the union of elements (such as body, emotions, thoughts, and sensations) that constitute the individuality and identity of a person" (MerriamWebster 2019). Mitchell (2005 - referring to Jung) maintained that "The self is not only the center, but also the whole circumference which embraces both conscious and unconscious; it is the center of this totality, just as the ego is the center of consciousness" (p. 4).

Considering this definition and concept and relating these with the developmental levels of the central neurological system, we can identify three interdependent and integrated Selves.

Ego states were described by Eric Berne (1972), "phenomenologically as a coherent system of feelings related to a given subject, and operationally as a set of coherent behavior patterns; or pragmatically as a system of feelings which motivates a related set of behavior patterns" (p.11). These behaviours were classified by Berne as Child ego state (Child), behaviours, thoughts and feelings related from childhood; Parent ego state (Parent) behaviours, thoughts and feelings copied from parents or parent figures and the Adult ego state (Adult) behaviours, thoughts and feelings which are direct responses to the here-here and-now. Therefore, we can conclude that the three ego states are not entities, nobody is in an ego state, rather they are expressing behaviour patterns classified as representing Child, Parent and Adult ego states, and that these are in effect sequentially developed.

The three different levels or Selves are the Physiological Hereditary Self (Natural Self),
Psychosocial Self (Social Self) and, the Conscious Self (Conscious Self). This article will explore how the different Selves originate behaviours that we can classify as ego states, their neurophysiological constitution and development, and the need to understand these to design adequate strategies and tactics to meet client contracts.

Sequentially, in terms of human development, the first Self to be developed is the Natural Self which initiates its development process at conception and continues as the autonomous nervous system completes its development (1 $1 \frac{1}{2}$ years of age) (Stiles and Jernigan, 2010). Using the information stored in its genetic memory (Cardinali 2001) from millions of years of genetic development and those more recent experiences of ancestors (Kandal, Schwartz and Jessel 1991), this Self's principal objective is survival, as a being and species.

The second Self to be formed Is the Social Self. This Self initiates its development as the cerebral cortex evolves and continues its development in different stages, adapting to significant changes in the environment (see Figure 1). Based on the phylogenetic hereditary memory relating survival to social integration and acceptance, this Self is developed to help us survive in the environment in which we live. Its development is initiated after birth and formed by verbal and nonverbal instructions received from the principal caretakers, who at this point have the survival of the new being in their hands.

The infant, aware of its dependency, considers that which is communicated to it, through actions, words, tutorials and modelling, to be essential to its personal and social survival. These learnings, stored in the ontogenetic memory, also register the emotional impact that this learning process has had (Stiles and Jernigan 2010; Li and Fan 2019; Thompson and Kim 1996).

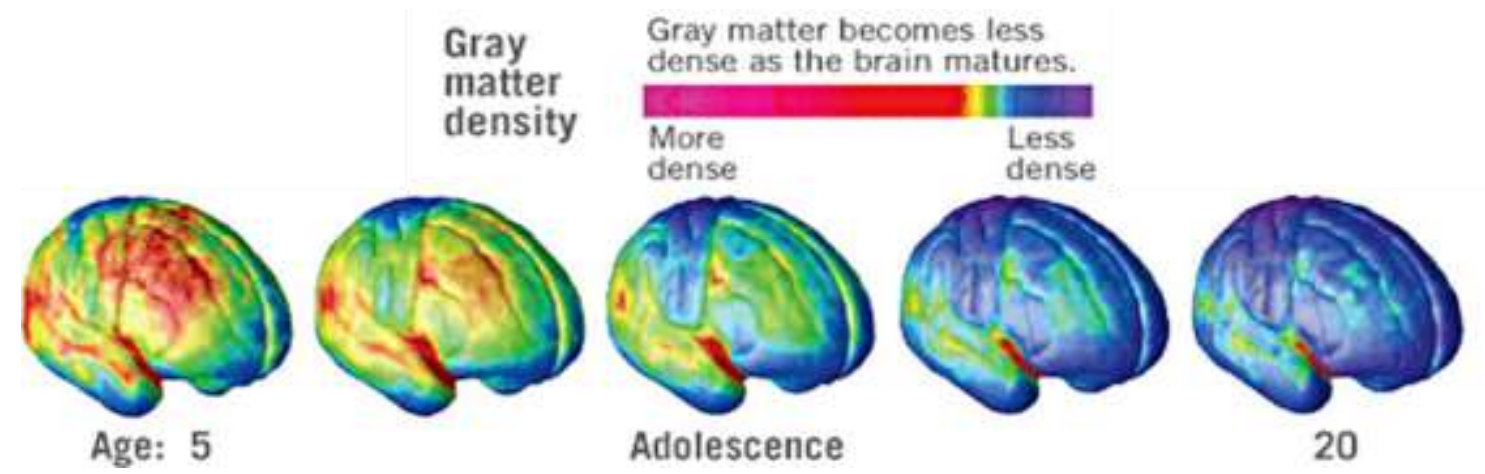

Figure 1: Stages of Development of the Social Self (based on material from Gogtay, N., Giedd, J. N., Lusk, L., Hayashi, K.M., Greenstein, D., Vaituzis, A.C., Nugent III, T.F., Herman, D.H., Clasen, L.S., Toga, A.W., Rapoport, J.L. and Thompson, T. M. 2004). 
The third Self, the Conscious Self, is that part of the human being that operates in the here-and-now, and is conscious of its Self, its surroundings and environment, its behaviour, the adequacy of the behaviour and the analytical processing of perceived stimuli, the interpretation of the stimulus and the adoption of adequate behaviour to meet needs. Its objective is to transcend animalistic beginnings, giving purpose and significance to Its existence. Present at conception and as the Social Self develops, it will be limited in its expression as the neurophysiological organs it requires to manifest are still in development (Superior Frontal Lobe). This Self starts interacting, reviewing, and evaluating the adequacy of the incorporated instructions at adolescence, when the frontal lobe starts to intensify its development, reaching full expression capabilities at early adulthood when fully developed (Tanaka, Matsui, Uematsu, Noguchi and Miyawaki 2012; Kandal et al 1991; Li and Fan 2019). This Self can assume the executive function of the personality.

\section{Behaviour}

An initial word about behaviour - all living things endeavour to maintain homeostasis; the lack of homeostasis generates needs (Cooper, 2006). The needs generated by the lack of homeostasis require their satisfaction to regain the equilibrium required for healthy well-being/survival. Whatever behaviour we initiate, be it drinking, playing, transacting or making strategic alliances, it is because we are subjectively feeling a certain need.

In each context or situation, a stimulus will be perceived by the senses and proprioceptive receptors that will be transmitted by the peripheral nervous system through the autonomic nervous system to the rest of the central nervous system (Waxenbaum and Varacallo, 2019). This implies that primitive hereditary behaviour will be initiated before automatic or voluntary behaviour. The resulting precept (general rule) will be analysed by the genetic/kinetic hereditary memory, initiating an interaction with the endocrine system resulting in the production of chemical substances that will create physical behavioural responses. The precept, continuing its advance through the central nervous system, may also initiate protaesthesia or conscious behaviour (Hall, 1998). In all instances, the resulting behaviour will be directed to fulfilling a latent need.

Adequate behaviour is that which satisfies a need directly; inadequate behaviour is that which does not satisfy the need, even if it pacifies it by producing a pleasurable response.

The Natural Self will receive first the stimulus (Cardinali, 2001) and will interpret it considering the information stored in the genetic memory, hereditary script, "life plan control" (Berne, 1961, p. 87) and will emit neurotransmitters to promote the physical reactions required to meet the latent need given the interpreted emotional scenario (Thompson and Kim, 1996). The Free Child ego state will then manifest behaviours that can be classified as emotions (fear, anger, joy, sadness, or libido), and adopt behaviours to meet the latent need, consistent with the perceived stimulus and resulting emotion, the internal physiological reactions (Li and Fan 2019).

The Social Self will receive the stimulus and process it considering the information stored in the ontogenetic intrinsic memory. This interpretation may stimulate a sensory-based experience resulting in primitive or archaic behaviour if the perceived stimulus triggers past experiences that conform to the intimate script "adaptations of infantile reactions and experience" (Berne, 1961, p. 117). The individual will utilise the stored information/behaviour and emotional content, re-living the past situation in the here-and-now. The Parent and Adapted Child ego states will manifest themselves as they did in the past even though the present situation is different.

As the Conscious Self continues its development stemming from the prosencephalon (forebrain) and the frontal lobe acquires functional capabilities, it can perceive and analyse the adequacy of the behaviours (Thompson and Kim, 1996; Kandal et al 1991; Fonagy 1996), both internal and external, and conclude as to their adequacy in the satisfaction of the latent need. If the conclusion is that the behaviour is inadequate it can promote the adoption of adequate behaviour.

\section{Composition of the Selves}

Each Self has its layer of physiological components, needs, and behaviours to meet them. Operating through different parts of the central neurological system, they are independent of one another, operating as interdependent parts of an indivisible system.

\section{The Natural Self}

The Natural Self is that part of the human being that is responsible for physiological survival and registers thousands of millions of years of learning derived through the accumulation of experiences from the different forms of life that preceded It. It is that part of the human being that operates automatically, creating, recreating, and operating cells, organs, and organ systems in such a way as to permit survival. It is that part of the central nervous system that will initiate behaviours, interacting with all the components of the central nervous system, producing psychosomatic effects, affecting both physiological and psychological homeostasis (Howard and Lewis, 1972).

The behaviours to meet latent needs are based on the interpretation of perceived stimuli, generate organic reactions, called emotions, and transform electric 
energy into chemical energy, and chemical energy into mechanical energy (Cardinali, 2001; Kandal et al 1991). Using the central nervous system's most archaic parts, it will be fully functional after the primitive archaic autonomic brain completes its development, initiated three weeks after conception and completed around 18 months after birth (Stiles and Jernigan, 2010). This area does not differentiate between imagination/fantasy and reality; for it everything is as if it were real - what psychological theorist Fonagy (2001) calls 'psychic equivalence'.

The 10 organ systems created and operated by it, that work with one another interdependently, are the integumentary system, skeletal system, muscular system, lymphatic (immune) system, respiratory system, digestive system, complete nervous system, endocrine system, cardiovascular system, and the reproductive system (see Figure 2).
The needs to be met to maintain the necessary homeostasis are in order of importance for survival, oxygen, thermal regulation, hydration, sleep, nutrition, excretion, sensory stimulation, physical activity, and emotional expression. The organic reactions that we call emotion are provoked by the autonomic nervous system, that interprets the stimulus and interacts with the endocrine system to produce neurotransmitters that induce the different organic systems to produce behaviours that we classify as fear (flight), anger (fight), sadness, joy, and libido. These are natural emotions; other emotions can be referenced but they are not a product of the autonomic nervous system.

The information required to complete its purpose is stored in the phylogenetic memory, genetic memory, and constitutes a nuclear hereditary script and that answers the question: 'What am I?' and 'What needs to happen to survive?' (see Figure 3).

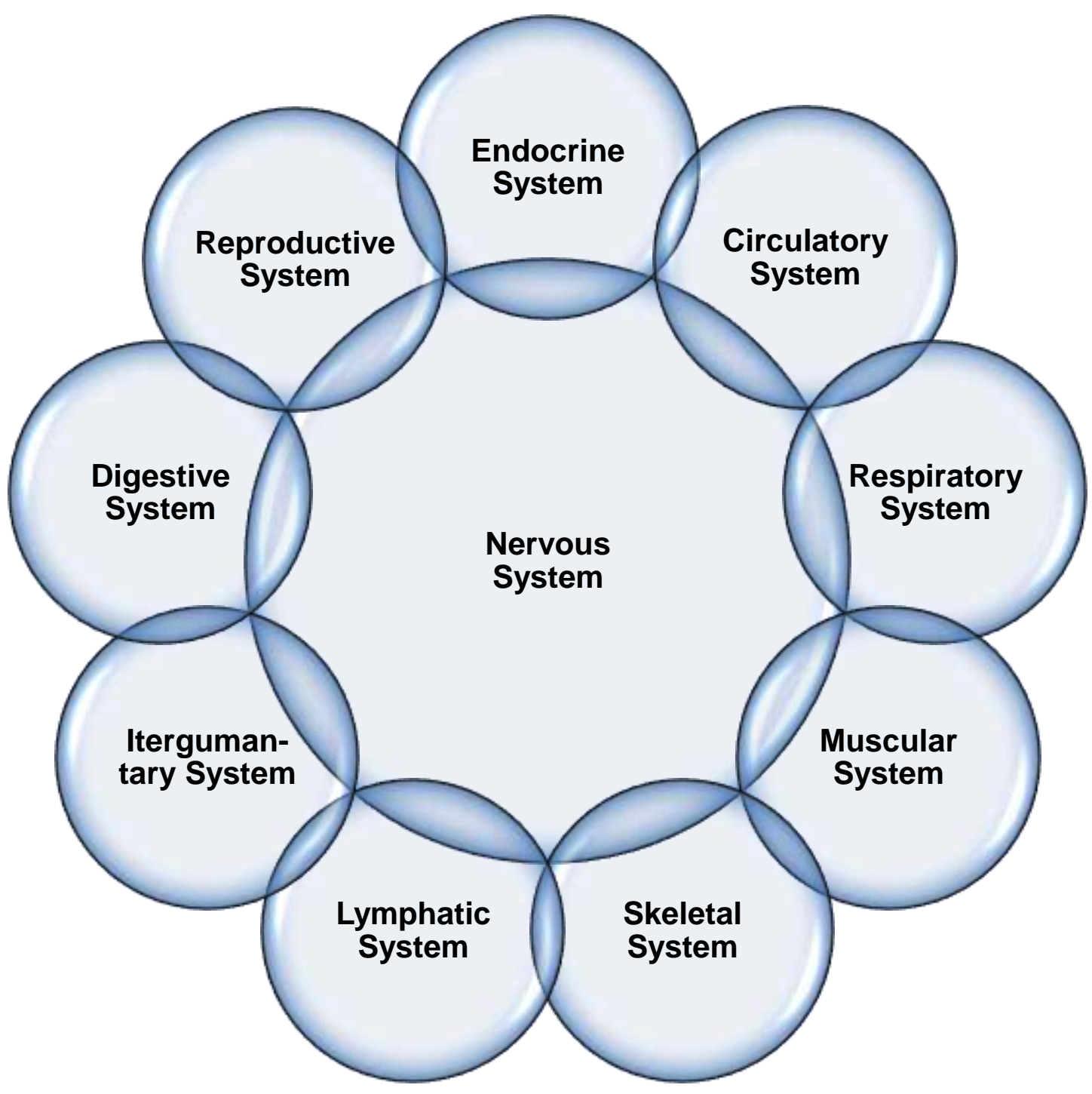

Figure 2: The Organ Systems 


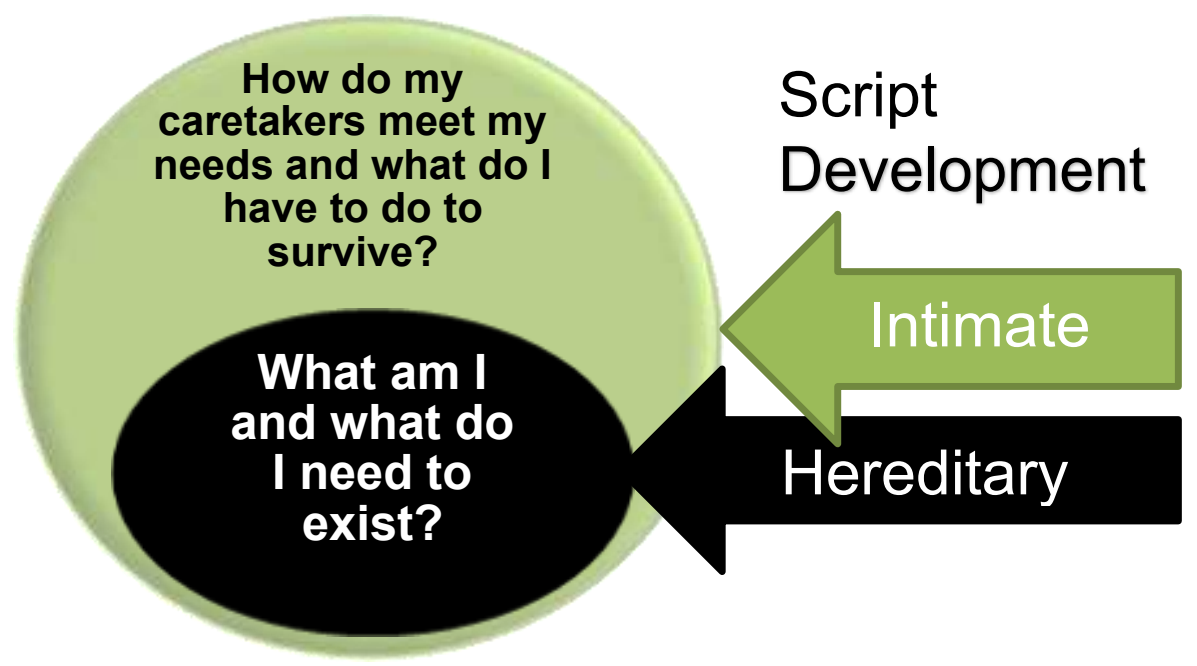

Figure 3: Development of the Intimate and Hereditary Scripts

The Social Self

The Social Self teaches us how to meet our needs in the environment we are inhabiting. Our Natural Self identifies the needs to be met; however it needs to learn how to meet these in the inhabited environment. It is the caregivers that indicate how to do so when attending to the child's needs (see Figure 4).

As individuals choosing behaviours, we are oriented toward seeking our well-being. Hence, the tendency to approach new behaviour in the hope of being rewarded by satisfying needs. However, in some instances our experience may have taught us that such pleasure-seeking behaviour might be punished. Our behavioural options include fight or flight, or inhibition of our behaviour and adoption of those imposed, and/or to go unnoticed, avoiding confront- ation. Adoption of imposed behaviours to eliminate dangers that are perceived to threaten integrity and well-being (Hall, 1998) are not without psychological effects, notwithstanding that this cost is minimised by the understanding that the affirmative action would bring about greater dangers.

The experienced instructions required to meet needs and protect existence, without suffering confrontation, are stored in the intrinsic (emotional) ontogenetic memory, that originates in the amygdala and involves the cerebellum and neocortex subsystems (Stiles and Jernigan, 2010). Learned instructions are stored in the explicit memory, that originates in the hippocampus and involves the medial temporal lobe subsystem ( $\mathrm{Li}$ and Fan 2019), that can be accessed cognitively.

6 yrs. Forebrain

3 yrs. primitive cortex

18 month old Temporal lobe

12 month old Diencephalon

7 month old mid Brain

3 month old hind Brain

At Birth Medulla, spinal cord

Figure 4: The Social Self

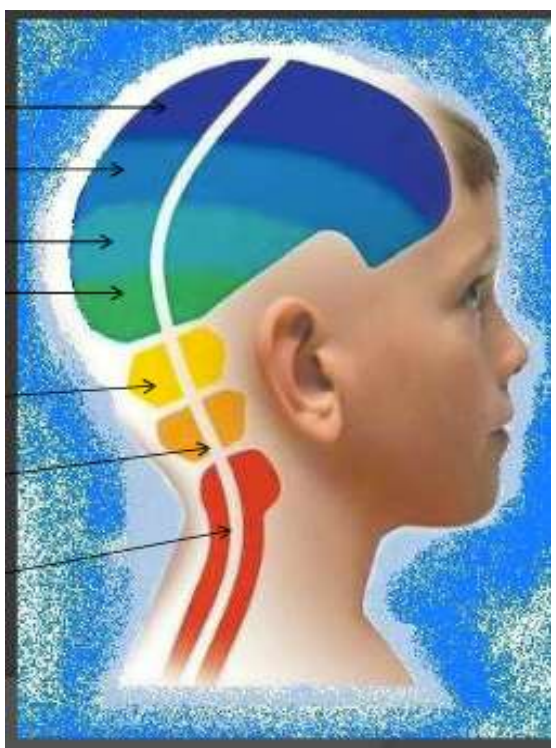


This indicates that, in all circumstances, the intrinsic memory is activated before the extrinsic memory. The extrinsic memory initiates its operations around 18 months after birth, as the brain develops, and both continue to operate through the lifetime. The behaviours and implicit values received and adopted to satisfy needs following the perception of the instructions received from and reinforced by principal caregivers, constitute the basis for conditioned behaviour (Waxenbaum and Varacallo,(2019) and the uncensored value system that may govern our behaviour (Tanaka et al 2012). A sensory pathway that runs through the central nucleus of the amygdala constitutes the basis for automatic reactions using the somatic sensory cortex of the parietal lobe in connection, basically, with the occipital lobe and the temporal lobe (Kandal et al 1991; Dias and Ressler, 2013; Thompson and Kim, 1996). This behaviour is based on past experiences and is independent of adequacy to the here-and-now.

The original hereditary script will now be enhanced with the information acquired that answers the questions 'Who do I have to be?' and 'How do I have to behave to be accepted and survive in this environment?' This information is stored in the intrinsic ontogenetic memory and will constitute the intimate script. To these two script modules, an additional mod- ule will be added consisting of those instructions stored in the extrinsic ontogenetic memory (see Figure 5). The needs to be met at this point in development are that of social integration (hierarchy and function), acceptance, protection, understanding and participation.

The emotion that this Self learns to express may originate from natural emotions and/or those learned to disguise them. Examples of learned emotions in our societies are resentment, anger, anxiety, confusion, jealousy, phobic fears, envy, anguish, irony, unloved, inadequate, shame, anxiety, guilt, depression.

Our behaviours, up to this point, are thus the product of these two influences: the way that evolution has structured our brains (the physiological Self) and the way that our environment individualises this structure throughout our lifetimes (the Social Self) (Cardinali, 2001).

\section{The Conscious Self}

The Conscious Self is that part of our being that operates in the here-and-now and can be used as our brain's executive. This Self looks to answer the question of 'Who Am I?' and 'What Can I Become?'

This Self refers to a full range of abilities including planning, organizing, goal setting, flexibility, selfregulation, inhibition and self-maintenance.

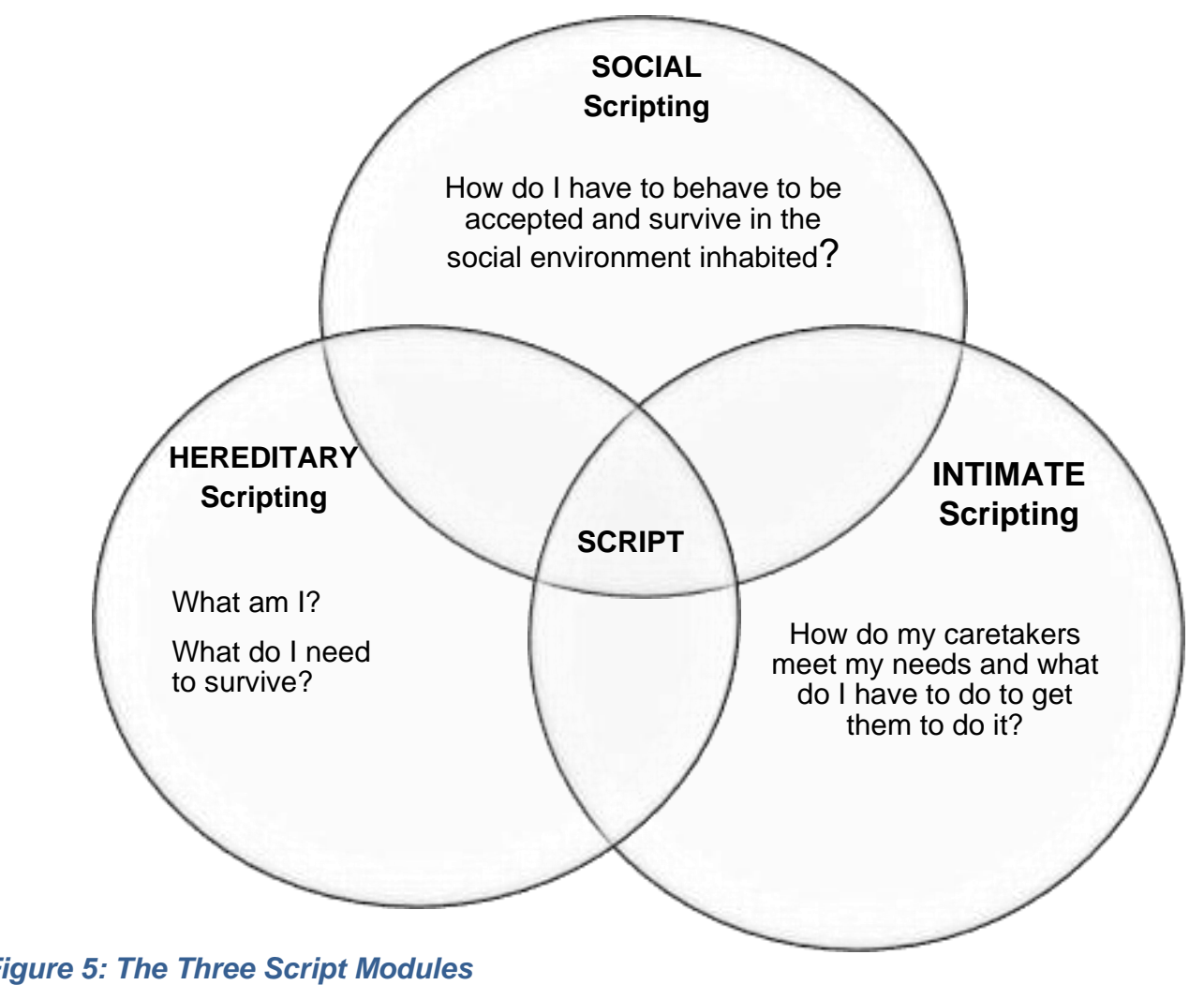

Figure 5: The Three Script Modules 
The functions performed by this Self are also those of the organization and control mechanisms of the persona. These cognitive processes are mediated by the frontal lobe. and as the frontal lobe completes its formation, around 19 years of age (Tanaka et al 2012), this Self will be able to express itself fully at this time.

Notwithstanding as the frontal lobe intensifies its development at adolescence (see Figure 6), the conscious Self starts to audit the values and behaviours incorporated and treatment received, based on caregivers' actions, as to their adequacy in satisfying the needs directly. This can manifest itself, at this time, by the disqualification of caregivers, redecision, and/or adoption of defence mechanisms that can be classified as pathology.

The needs that arise, as this Self reaches full expression, are to give significance and purpose to its existence, intellectual and ethical development, and the need to love and be loved.

This Self expresses sentiment such as empathy, selfesteem, happiness, compassion, love. Not expressing emotions, however, it can give information, protection, and permission to assist the adequate expression of natural emotions by the Natural and Social Selves, and neutralise inadequate learned emotions and the resulting chronic stress.

\section{Formation of Ego States}

Ego state behaviours are expressions of the reactions to perceived stimuli as interpreted by the central neural system (Llinás, 2001) to maintain physiological and psychological homeostasis, meeting needs. Note that the reactions will depend on the degree of development and adequacy of the organs and other physiological components.

The formation and evolution of the ego states are therefore intimately related to the formation and evolution of the Selves and central neural system and the environment in which they occur. It is also interesting to understand the distinction made between structure and function.
Development of Structure and Function of the Child Ego State

Structurally, the behaviours that constitute the Child ego state originate in the Natural Self. It will, therefore, contain autonomic involuntary and voluntary behaviours. The visible clues as to which ego state is operating are words, tones of voice, gestures, postures, and facial expressions (Berne, 1961).

The autonomic involuntary behaviours are not always visible, but they can be perceived by the physiological effects caused. The transmission of impulses through the peripheral nervous system to the central nervous system may not be visible, and the interpretation of these stimuli and the emission of neurotransmitters to promote organic reactions to the stimulus may also not be visible. However, the physiological implications of the organic reaction may be measurable and visible.

Two simple examples. We may not see the neural system telling the heart to beat at a certain level, yet we can measure the heart beats per minute. It is not readily visible, either, when the amygdala receives and interprets a stimulus and communicates with the hypothalamus, that then sends messages to the different glands and organs to produce certain hormones that provoke organic reactions. However, we can see the effects in facial expressions, gestures, posture and tones of voice that can be classified as emotions.

Therefore, as invisible as it may be, the Free Child or Natural Child will be structurally present, directly or indirectly, in all behaviour.

Functionally the Adapted Child is developed and functions independently of the Free Child and is the effect of the Social Self (Parent ego state). In other words, the principal caretakers indication of the 'acceptable' behaviours and implicit values to use to meet the individual's needs, and the individual's interpretation of the insistence that these behaviours be used, create the adapted behaviours that compose the Adapted Child ego state, as a direct result of Parental influence.

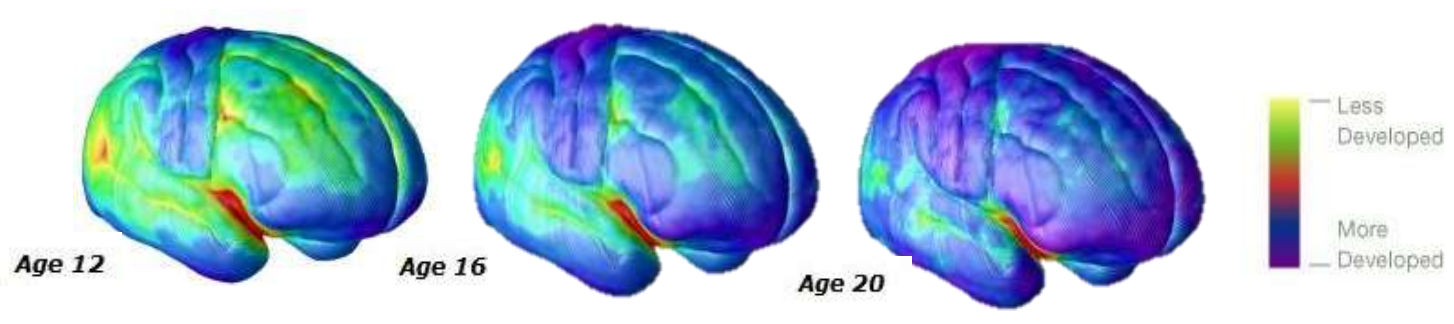

Figure 6: Stages of the Conscious Self (based on Gogtay et al, 2004 ) 
As we will see in the next section, it is my belief the Adapted Child is best expressed as part of the Parent ego state and not part of the Child ego state.

\section{Development of Structure and Function of the Parent Ego State}

Structurally the behaviours that constitute the Parent ego state originate in the Social Self. Typically, the Parent ego state will start manifesting itself towards the end of early childhood, around 18 months of age, and will be intensely present after that. The behaviours manifested and classified as being those of the Adapted Child are automatic conditioned responses to the perceived stimulus as interpreted by the Social Self's neurological circuits. The interpretation, as indicated above, in the corresponding section, is based, in infancy, on the reactions and values expressed by the caregivers, interpreted and stored in the corresponding intrinsic memory. They are, therefore, triggered as an automatic response to meet needs, that were registered as the accepted behaviours that would not produce confrontation and the consequential negative effects.

The Parent Self stores the values, actions and information communicated by the caregivers as interpreted by the individual. These constitute the basis for social survival in the environment in which the individual operates. Based on the interpretation of stimuli, these values and information will trigger the stored conditioned behaviours, and the Adapted Child appears.

We can, therefore, conclude that the Parent ego state creates the Adapted Child and is the trigger for automatic adapted conditioned behaviour. Figure 7 represents the formation of the Parent, and Adapted Child ego states.
Development of Structure and Function of the Adult Ego State

Although present at birth, the Conscious Self that originates the behaviours classified as being those of the Adult ego state will become more visible in adolescence and as the individual strives to break the primary symbiosis and acquire the information to operate in the here-and-now on their own. This ego state can be predominant and govern the individual's behaviour (see the executive brain function at Figure 8).

The behaviours of the Adult ego state are those that distinguish us from the rest of the animal kingdom. This ego state is the essence of the individual being. It can supervise the functioning of the Free Child ego state, filtering and retaining the adequate behaviours required for its survival, changing forms of meeting basic needs so that they can be satisfied directly.

It can review adapted conditioned behaviours to identify the adequacy of these to the here-and-now, adjusting them and/or adopting them as being adequate. It can review caregiver guidelines, values, actions, expectations, and permissions or limitations. It can adjust them to realities of the here-and-now and retain those that are consistent and adequate for the individual's healthy well-being.

The Adult ego state, based on the Conscious Self, is, therefore, the true expression of the individual's Being. It constitutes a summary of the other ego states, as analysed, filtering, and adjusting them to meet needs directly in the here-and-now. This form of viewing the Adult ego state is registered as being that of the Integrated Adult.

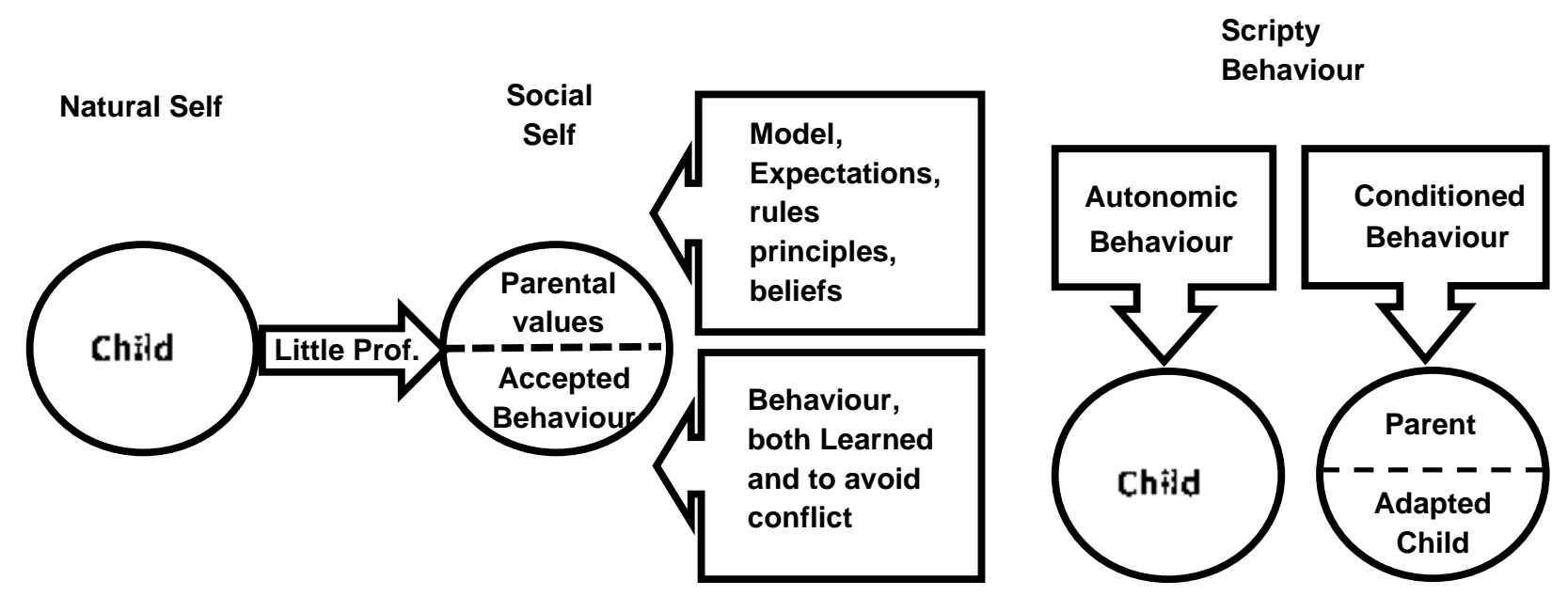

Figure 7: How Parent creates Adapted Child 


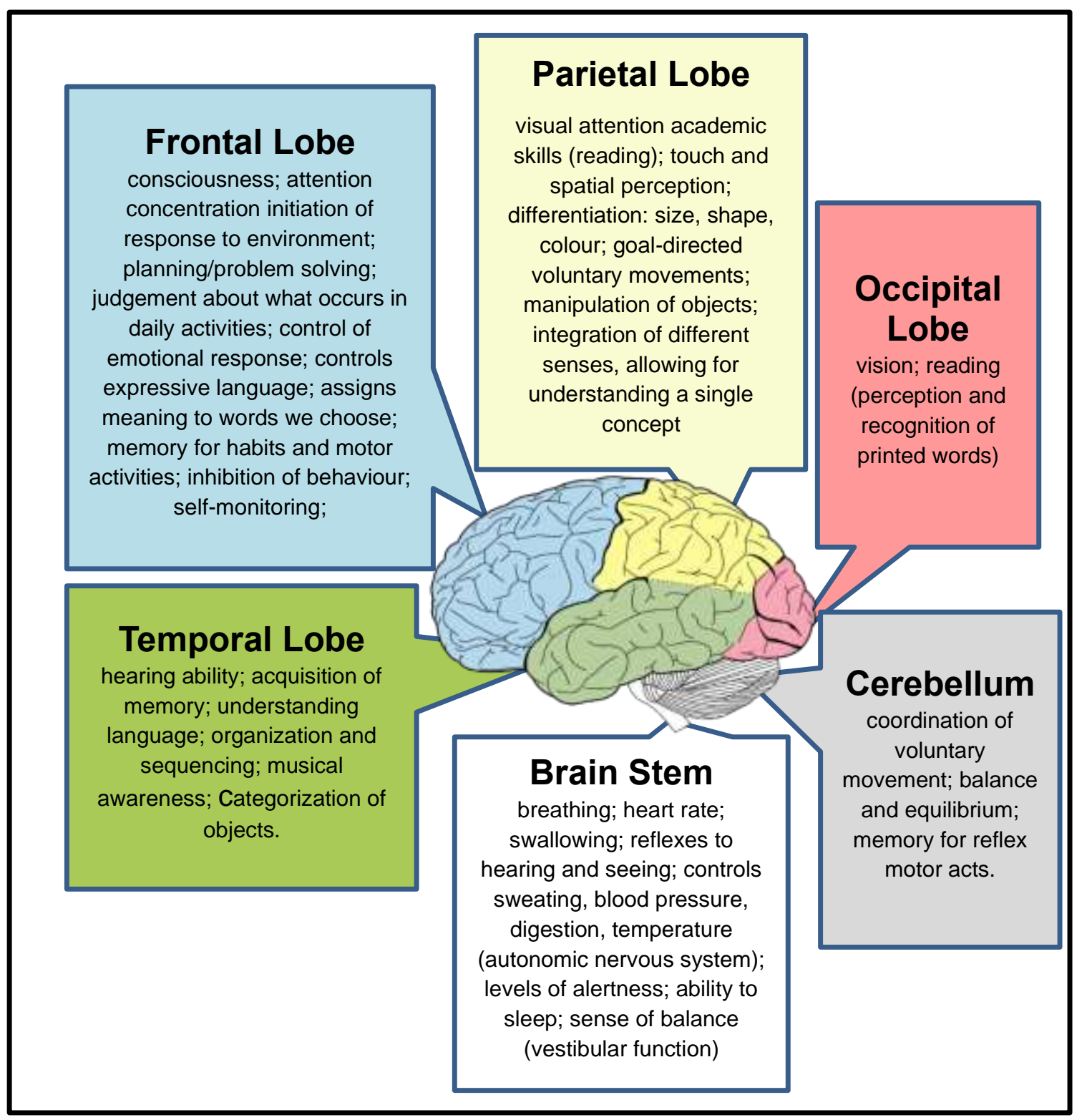

Figure 8: Executive Brain Function

Considering that this ego state's neurophysiological behaviour originates from the interactions between the superior frontal lobe, inferior frontal lobe, parietal lobe, occipital lobe, temporal lobe, and their specific functions, it is difficult to view a structural Adult ego state that is not Integrated (see Figure 4). Adult behaviour is based on proprioception of Free Child autonomic behaviour (pathos), reviewed and accepted Parent ego state information and Adapted Child behaviours (ethos), and superior analytical capacities based on sustainable information (technos). These integrated behaviours are present even in early childhood, notwithstanding they will become evident and accessible in adulthood, as the frontal lobe reaches maturity.
Ego State Contamination and Decontamination The following section is based on over 40 years of my working with clients. It reflects the conclusion I have reached for what contamination is, how it works to reinforce scripts, and what decontamination is all about. Considering the neurophysiological components involved and their interaction, contamination is always double.

Theoretically, I understand the possibility that contamination, considering the definition of ego states, could be considered to occur as one ego state contaminating the Adult; however, the behaviour produced by contamination indicates that all three ego states are involved. 
Contamination is initiated by Parental interpretation of a here-and-now stimulus, generating a delusion, that triggers an Adapted Child illusion, based on past experiences, to be dangerous for its well-being. The Natural Child experiences this imagined threat as though it were real (psychic equivalence), and hence activates fight or flight mechanisms. The intensity of the situation absorbs psychic energy, limiting the Adult ego state's intervention. The Adult ego state is seen as being contaminated when in reality it is not being activated and left with little psychic energy.

As the situation develops and the Adapted Child reacts with an inadequate behaviour to the here-and-now, the results will end up confirming the parental injunctions and therefore advancing the script. This is because delirium creates phobic reactions that trigger emotional responses through psychic equivalence that inhibit here-and-now adult behaviour (see Figure 9).

When we decontaminate, we use the Adult ego state's executive function to promote the liberation of the Natural Child and supervise the adequacy of its behaviours to be acceptable in the context in which they occur. We also promote the adequacy of the Parent to the here-and-now, so that its value system and the behaviours it allows and sponsors will contribute to the direct satisfaction of latent needs. It becomes evident that the Parent is the contaminator, that triggers the Adapted Child's fantasies that inhibit the Natural Child from operating adequately and introduces injunction-based information in the Adult. Thus, contamination is always double.

The implications are that we intervene in the Social Self to be able to permit the Natural Self and the executive Conscious Self to operate correctly.

\section{Script formation}

I believe that the dominating effect that scripts have on people's lives is because they are life plans that are established to permit the survival of the individual.

As scripts are based on trial-and-error experimentation, be it hereditary or present life experiences, they operate on an autonomic and/or conditioned basis. Therefore, a hereditary script initiates at conception (to permit existence) and continues to be developed throughout the individual's life (to permit survival).

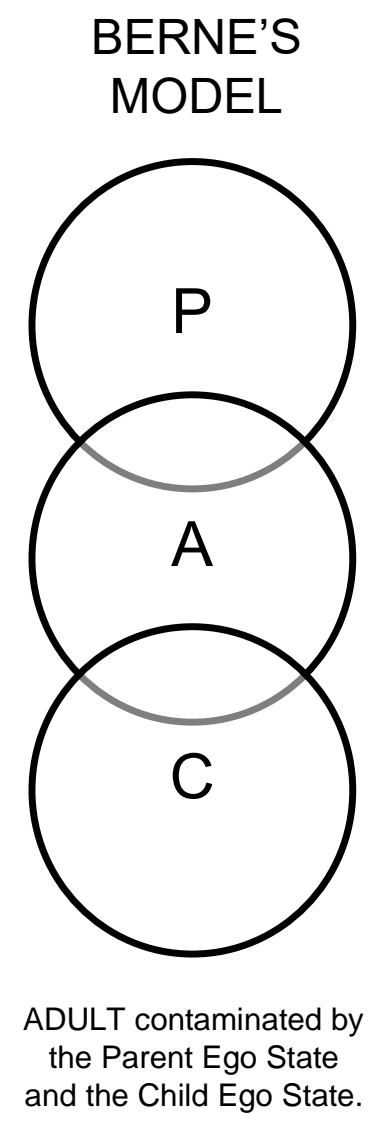

SUGGESTED MODEL

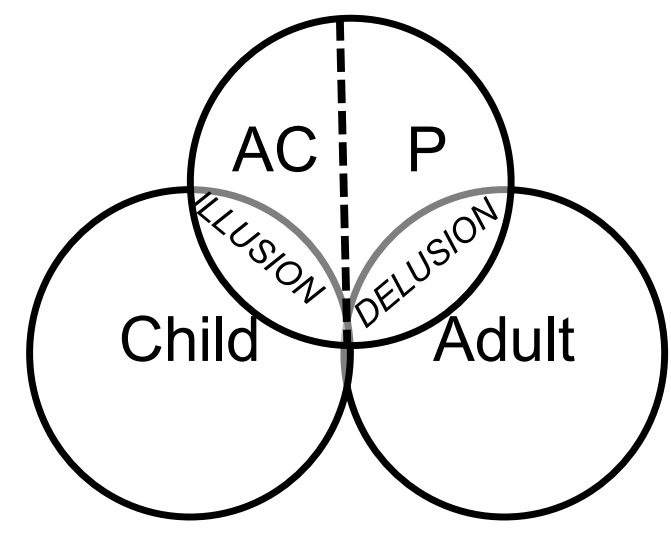

PARENT Injunctions generate Adapted Child Delusions that trigger Natural Child Emotions Inhibiting Adult intervention. 
Scripts contain the set of actions, values, principles, beliefs (about us and others), and basic norms of behaviour to be taken to meet needs, to meet expectations and to survive in the environment we inhabit.

They are the 'rules' that must be followed and adopted as guidelines and premises to govern our existence. These rules may be adequate or Inadequate. They are adequate when meeting needs directly and Inadequate when not meeting them. They may also be appropriate or inappropriate depending on the context within which they occur.

These rules are stored in different memories, at different times in the individual's development process. The memories involved have different characteristics and are independent of each other. Each uses different neurophysiological areas and has unique functional capabilities and accessibilities. Therefore, to access and/or intervene in these memories different techniques and methods need to be used.

This being the case I will now identify the significant script developmental instances and the memories involved, based on the physiological development of the central nervous system:

1. Hereditary Script answers the question 'What am I?' and 'What do I need to exist?' and is transmitted, incorporated, and stored in the genetic memory. This script initiates its expression at conception and is used to form the individual, organizing the cellular growth and structuring of the different organs and systems that will permit development and survival. It will also be responsible for physiological homeostasis and adequate functioning of the Natural Self. The contents of this script tend to be autonomic and involuntary. To access this memory specially designed multi-facet strategies and methods may be required, involving physiological, kinetic, pharmacological, medical, and sensory actions.

2. Intimate Script answers the question of 'What must I do to survive?' Its formation is initiated around 18 months after birth, when the superior brain formation is complete. As indicated in the corresponding section referring to the formation of the Social Self, this script is stored in the intrinsic ontogenetic memory and contains the actions required to survive in the intimate environment to which it is tied. It will continue to incorporate traumatic experiences that the individual identifies as life-threatening (psychic equivalence). This memory is activated by the perception of stimuli that trigger the memory of the situation lived, along with its emotional content. It can be accessed by the interpretation of stimuli. The cont- ent of the script tends to compel the individual towards automatic compulsive adapted behaviours identified to avoid conflicts and protect the self.

3. Personal Script (ages 3 to 9 years) answers the question of 'Who do I have to be, to be okay, and accepted in my intimate nuclei?' The content of the script is stored in the intrinsic, if traumatic, and extrinsic ontogenetic memory. It is designed to protect the individual as described in the section referring to the Social Self and constitutes what may be called the personality. This memory can be accessed cognitively. The contents of this script, although leading to compulsive behaviours, can be accessed consciously.

4. Social Script (ages 9 to 17 years) answers the question 'What is my social identity, hierarchy, and what is expected of me?' This content is stored in the extrinsic ontogenetic memory and can be accessed biographically.

5. Geopolitical Script (19 plus years old) answers the question 'What is my place in the world and what do I need to succeed?' (economically, politically, geographically, ideologically, religiously). The content is incorporated into the extrinsic ontogenetic memory. It can be accessed biologically and tends to be conscious behaviour.

Figure 10 represents an individual script with its components: (the lines separating the different script segments indicate the permeability and facility in accessing).

\section{Script Intervention Strategies}

Script intervention strategies require identification of the segment in which originates the behaviour which is contracted to be adopted, neutralised, or modified, and which other segments are affected. It has been my experience that the cause will reside in one segment but the effects will manifest themselves in one or more segments. The importance of this understanding resides in the fact that the methods appropriate to be effective in intervention of the different segments is rarely the same in procedure, quality and intensity.

For example, if the contract is limited to modifying a specific behaviour (effect) then we can work within a given method. However, if the contract is to eliminate or neutralise negative script effects on the client's healthy well-being, then we will need to identify where the cause resides and not limit ourselves to working with the manifested behaviours. The latter will then require, on our part, the ability to work with different methods, formulating an intervention strategy that will integrate different procedures and may require the intervention of different professionals with specific skills, as will become evident in the following examples. 


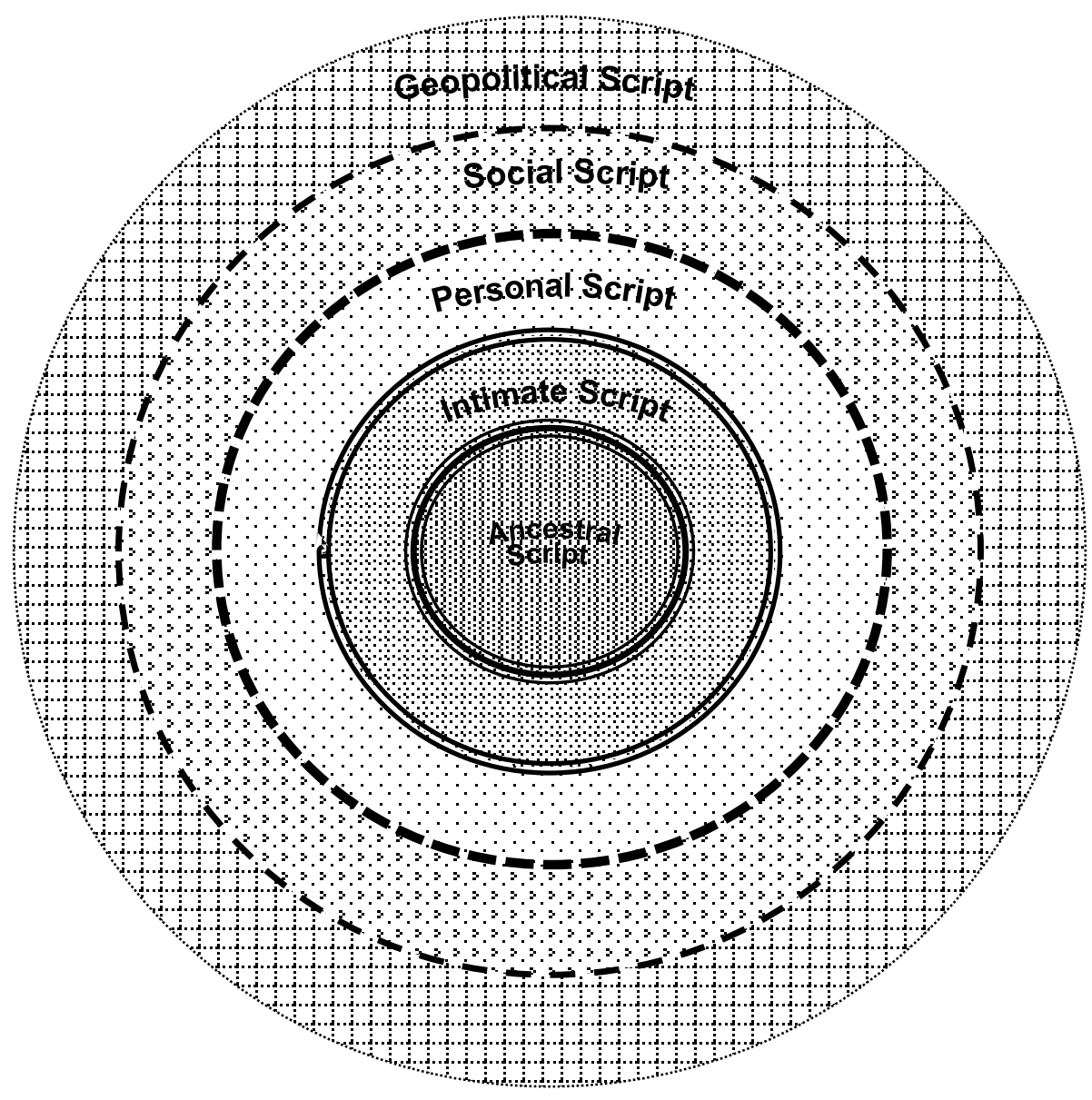

Figure 10: The Components of an Individual Script

If in the Hereditary Script (genetic memory), the methods chosen to change behaviours can be related to medical, psychiatric (medication that inhibits the production/absorption of neurotransmitters and/or promotes them), physiotherapeutic methods such as EMDR (Shapiro, 2001) that use sensory stimulation can activate primal instructions/memories, to be cognitively managed with TA, musicotherapy, bioenergetic, nutritional, etc.

It is important to note that the autonomic brain does not distinguish between reality and fantasy. Therefore, emotional physiological responses to perceived stimuli originating from other script segments can and will activate involvement of this segment, causing involuntary behaviours and affecting the physiological healthy well-being of the client (psychosomatic influence).

Using stress as an example: healthy stress is shortlived, initiating when a stimulus is transmitted through the central nervous system and is interpreted by the autonomous brain as indicating that the individual is in harm's way. Our autonomic brain prepares our organism for flight or defence (fear/anger), thus activating the sympathetic nervous system by liberating the neurotransmitters required to produce the physiological responses necessary for protection and survival. When the cause for the existence of the stimulus ceases, a new stimulus is transmitted indicating that the individual is no longer in harm's way and our organism returns to its normal functioning.

Chronic stress is long-lasting, when the perceived stimulus activates a latent need and is interpreted, based on past experiences in other script segments, as being harmful to the individual's psychological healthy well-being. It interacts with the autonomous nervous system indicating that the individual is in danger. The autonomous nervous system activates its natural procedures to defend the individual's survival. The problem arises when the individual does not implement the physiological responses for which the hormones were liberated. As the mechanical effects required (flee or fight) are not being used to eliminate the hormones, they then continue to influence the organs and organ systems so as to inhibit their normal functioning. This will bring about negative physiological effects. 
Considering the stress examples given above, if the patient were to be treated for high blood pressure caused by healthy stress, through medication in a short time he would be returned to a healthy situation. However, if chronic stress caused the situation, the medication would be reducing the effects and not eliminating the cause and will therefore not be resolving the situation but only mitigating it.

It is evident that when we want to resolve a situation, we need to identify the cause. Is it a physiological homeostatic or a psychological homeostatic? The first will be indicative of the hereditary script segment and the second would be indicative of another segment.

If the Intimate Script (intrinsic unconscious memory) is involved, the method chosen requires the identification of the stimulus that trigger the undesired behaviour and to duplicate it in controlled circumstances, in order to be able to neutralise the emotional impact and permit the identification of adequate behaviour that can substitute it and be conditioned in its place. In my experience EMDR, developed by Shapiro (2001), together with cognitive assertive TA, has proven to be effective. Also, Gestalt therapy (Perls 1969) together with TA using flexible methods is proven to be quite effective.

Personal Script (extrinsic preconscious memory) may respond to cognitive psychotherapeutic methods, and especially TA in its many facets can be effective.

If the Social Self (extrinsic biographical memory) is involved, cognitive TA counselling methods can be applied.

Finally, if the Geopolitical Script (extrinsic conscious memory) is active, TA coaching methods are appropriate.

It also seems that to be effective, multidisciplinary methods and models should be developed and used. Considering that professionals have their own specific and special capabilities with certain techniques, procedures or models, it Is important to be able to integrate with professionals of different specialties to be able to contribute to the fulfilment of our client contracts.

\section{Conclusions}

Based on the content in the body of this article we can conclude that:

- All behaviour is significant and directed to the satisfaction of a latent need.

- Behaviour should be classified as adequate when the behaviour satisfies the need directly, or inadequate when the behaviour does not meet the need directly even if producing a pleasurable experience.
- Ego states are useful tools to be used with clients, as Eric Berne indicated. However, practitioners need to understand the neurophysiological interactions that produce the behaviours, to be able to intervene in identifying causes from effects, and the elaborate strategies and tactics to meet contracted behaviours.

- The Physiological Self (Natural Child) is present in all behaviour on a 24/7, 365-day basis.

- The Integrated Adult ego state, representing the conscious self, is the executive power required to implement and support all forms of intervention.

- $\quad$ Scripts are necessary to survive, are continuously formed and adjusted, and can be divided into instances for intervention purposes.

This article identified the physiological, neurological and psychological determinants that arise from constraints imposed by both genetic and environmental factors, originating human behaviour.

The determinants, called Ego Selves, that organise the phenomena that Eric Berne classified, structured, conceptualised, and defined to mould transactional analysis and design instruments to assist professionals and clients to adjust behaviour, have also been analysed.

A different form of presenting the Adapted Child, differentiating it from Berne's model where the Adapted Child is shown as a part of the Child, was presented. Parent-Adapted Child, and the Adult ego states, adjusting their manifestation and organization to the physiological development of their corresponding ego self, were identified, proposing that the Adapted Child is a part of the Parent ego state.

Contamination was reviewed and adjusted for consistency between cause and effect. I propose that the contaminating ego state is the Parent ego state that is creating a delusion based on injunctions and this generates an illusion in the Adapted Child portion of it which in turn causes the Natural Child's emotional reaction, considerably limiting the Adult ego states capabilities to intervene.

Script analysis was reviewed and organised, indicating that the script is a life plan initiated at conception and ends at death. It is indispensable for survival, having adequate and inadequate segments that may limit lifespan and quality of life. Occurrences, neurophysiological factors, and memories involved in their development and implementation were also identified.

Suggestions and examples for the integrated development of intervention strategies and tactics to adjust behaviours and fulfil contracts were presented in the corresponding section. 
Finally, when we feel, our primal natural self (body), based on the past, is active; when we think, our conscious self (brain), in the here-and-now, is active. Our art, as practitioners, is to integrate the two to promote pleasure, well-being and happiness.

We need to remember that pleasure is obtained by meeting our body's needs, well-being is obtained by meeting our social need,s and happiness arises when our conscious needs are met.

Jorge Alberto Close is a Teaching and Supervising Transactional Analyst (Organisational, Educational) (with ITAA) and is also qualified by the Brazilian TA Association (UNAT) and the South American TA Association (ALAT). He lives in Argentina and can be contacted on closejorge@gmail.com

\section{References}

Berne, E. (1961). Transactional Analysis in psychotherapy. Grove Press.

Berne, E. (1972). What Do You Say After You Say Hello. Grove Press.

Cardinali, D. (2001). Manual de Neurofisiologia (8 Edicion). Juan Bravo.

Cooper, S. J. (2008). From Claude Bernard to Walter Cannon. Emergence of the concept of homeostasis. Appetite, 51(3), 419-427. https://doi.org/10.1016/j.appet.2008.06.005

Dias, B. G., \& Ressler, K. J. (2013-12-01). Parental olfactory experience influences behavior and neural structure in subsequent generations. Nature Neuroscience, 17(1), 96. https://doi.org/10.1038/nn.3594

Fonagy, P. (1996). Attachment, the development of the self, and its pathology in personality disorders.

Psychomedia, 26-32.

http://www.psychomedia.it/pm/modther/probpsiter/fon agy-2.htm

Gogtay, N., Giedd, J. N., Lusk, L., Hayashi, K. M., Greenstein, D., Vaituzis, A.C., Nugent III, T. F., Herman, D. H., Clasen, L. S., Toga, A.W., Rapoport, J. L. \& Thompson, P. M. (2004). Dynamic mapping of human cortical development during childhood through early adulthood. Proceedings of the National Academy of Sciences of the United States of America (PNAS) 101(21), 8174-8179.

https://doi.org/10.1073/pnas.0402680101
Hall, R. (1998). Neurological Basis of Classical Conditioning.

https://web.mst.edu/ rhall/neuroscience/05 simple I earning/classical.pdf

Howard, H. R., \& Lewis, M. E. (1972).

Psychosomatics- How your emotions can damage your health. The Viking Press Inc.

Kandal, E., Schwartz, J. H., \& Jessell, T. M. (1991). Principals of Neural Science (3rd ed.). Elsevier Inc.

Li, H., \& Fan, Y. (2019). Interpretable, highly accurate brain decoding of subtly distinct brain states from functional MRI using intrinsic functional networks and long short-term memory recurrent neural networks. Neuroimage, 202, 116059. https://doi.org/10.1016/j.neuroimage.2019.116059

Llinás, R. R. (2001). I of the vortex: from neurons to Self (pp. 190-191). MIT Press.

Merriam-Webster. (2019). https://www.merriamwebster.com/

Mitchell, G. (2005). Carl Jung \& Analytical

Psychology, the way to Individuation

https://trans4mind.com/mind-development/jung.html

Perls, F. (1969). Ego, Hunger and Aggression: The Beginning of Gestalt Therapy. Random House.

Shapiro, F. (2001). Eye Movement Desensitization and Reprocessing (EMDR) Therapy. Basic Principles, Protocols, and Procedures (Third Edition:). Guildford Press.

Stiles, J., \& Jernigan, T. L. (2010-11-03). The Basics of Brain Development. Neuropsychology Review, 20(4), 327-348. https://doi.org/10.1007/s11065-010$\underline{9148-4}$

Tanaka, C., Matsui, M., Uematsu, A., Noguchi, K., \& Miyawaki, T. (2012-12-21). Developmental Trajectories of the Fronto-Temporal Lobes from Infancy to Early Adulthood in Healthy Individuals. Developmental Neuroscience, 34(6), 477-487. https://doi.org/10.1159/000345152

Thompson, R. F., \& Kim, J. J. (1966). Memory systems in the brain and localization of a memory. Proceedings of the National Academy of Sciences, 93(24), 13438-13444.

https://doi.org/10.1073/pnas.93.24.13438

Waxenbaum, J. A., \& Varacallo, M. (2019). Anatomy, Autonomic Nervous System. Stat Pearls Publishing LLC. 